\title{
Herzinsuffizienz
}

\section{Nach Myokardinfarkt das schwache Herz besser schützen}

Häufig zeigen sich bald nach einem akuten Myokardinfarkt klinische Zeichen der Herzinsuffizienz. Ein längerer Klinikaufenthalt lässt sich bei entsprechender Medikation in Grenzen halten.

Etwa jeder dritte Infarktpatient zeigt bei der Notaufnahme klinische Zeichen der Herzschwäche - mit einem besonders hohen Risiko, in den nächsten Wochen oder Monaten zu sterben oder erneut stationär behandelt werden zu müssen. Die EPHESUS-Studie zeigt, dass die Therapie mit Eplerenon (Inspra ${ }^{\circledR}$ ) zusätzlich zu einer Standardtherapie mit ACE-Hemmern, Betablockern, ASS und Statinen die Überlebenschancen dieser Patienten erheblich verbessern kann [1].
An der randomisierten, placebokontrollierten Doppelblindstudie hatten 6632 Patienten mit klinischen Zeichen einer Herzinsuffizienz in den ersten Tagen nach einem Myokardinfarkt teilgenommen. In der Eplerenongruppe war innerhalb der ersten 30 Tage die Gesamtmortalität um $31 \%$ reduziert, die kardiovaskuläre Sterblichkeit um 32\% und die Sterberate am plötzlichen Herztod sogar um 37\%. Nach 16 Monaten lag die Gesamtmortalität in der Eplerenongruppe um 15\% niedriger als in der Kontrollgruppe [1]. Die Rate der plötzlichen Herztode war um 21\% und die Rate erneuter stationärer Behandlungen wegen Herzinsuffizienz um 23\% reduziert.

Eine Subgruppenanalyse zeigte zudem, dass Eplerenon die wegen einer Verschlech- terung der kardialen Pumpfunktion notwendigen erneuten stationären Aufenthalte im Mittel um 1,6 Tage verkürzen kann (9,2 vs. 10,8 Tage unter Placebo zusätzlich zur Standardtherapie, $p=0,019$ ). Insgesamt verbrachten die zusätzlich mit Eplerenon behandelten Patienten während des Follow-up-Zeitraums von 16 Monaten 3,6 Tage weniger zur Behandlung von Herzinsuffizienzsymptomen im Krankenhaus (13,3 vs. 16,9 Tage; $p=0,0006)$. Dieser Vorteil der Eplerenontherapie war weltweit in allen untersuchten Regionen vergleichbar [2].

(VS) II

II 1. Pitt B et al. N Engl J Med 2003;348: 130921; 2. Gheorghiade M et al. Am Heart J. 2009;158:43743; Pressemitteilung Pfizer

\section{Gliptin statt Sulfonylharnstoff \\ Langfristig gleich wirksam, aber weniger riskant}

Der DPP-4-Hemmer Sitagliptin schneidet hinsichtlich der Wirksamkeit und Verträglichkeit im Vergleich zu einem Sulfonylharnstoff auch im Langzeitverlauf gut $a b$. Das haben eine aktuelle 2-Jahres-Studie und eine ergänzende Post-hoc-Analyse ergeben.

Die Studie mit 1172 Typ-2-Diabetikern war auf zwei Jahre angelegt und zunächst dafür konzipiert, anhand des $\mathrm{HbA}_{1 c}$ die Langzeiteffizienz von Sitagliptin (Januvia ${ }^{\circledR}$, als Fixkombination mit Metformin Janumet ${ }^{\circledR}$ ) zu prüfen. Dafür erhielten die mit $1500 \mathrm{mg}$ Metformin oder mehr unzureichend eingestellten Patienten randomisiert zusätzlich $100 \mathrm{mg} / \mathrm{d}$ Sitagliptin oder den Sulfonylharnstoff (SU) Glipizid in einer Dosis von 5-20 mg/d. Nach 104 Wochen war die $\mathrm{HbA}_{1 c}-\mathrm{Ab}$ - senkung vom Ausgangswert mit beiden Präparaten ähnlich (-0,54 unter Sitagliptin vs. - $0,51 \%$-Punkte unter Glipizid). Nachdem der $\mathrm{HbA}_{1 \mathrm{c}}$ nach 24 Wochen seinen Tiefpunkt erreicht hatte, stieg er zwar im Rahmen des üblichen Fortschreitens der Diabeteserkrankung mit beiden Medikationen wieder etwas an. Der Anstieg fiel mit dem Gliptin jedoch geringer aus (16 vs. 21\%/Jahr).

Von Hypoglykämien berichteten unter dem Gliptin 5\% und unter dem SU 33\% der Patienten. Die Gewichtsentwicklung betrug unter dem Gliptin $-1,6 \mathrm{~kg}$ und unter dem SU +o,7 kg. Belastungstests dokumentierten zudem eine stabile Betazellfunktion mit Sitagliptin, die mit dem SU dagegen nachgelassen hatte.

Prof. Michael Nauck aus Bad Lauterberg betonte anhand der Daten dieser 2-Jahres-
Studie, dass SU ein nicht zu unterschätzendes Gefahrenpotenzial haben. Dieses äußere sich natürlich in Form von vermehrten Hypoglykämien. Zudem gebe es auch Hinweise, dass SU die kardiale Funktion beeinträchtigen und so vielleicht kardiovaskuläre Ereignisse begünstigen könnten. Für DPP-4-Hemmer sei dagegen ein entgegengesetzter Effekt erkennbar. Nauck: ,Warum also sollte ich einen Wirkstoff mit derartigen Nachteilen wie Sulfonylharnsstoffe verordnen, wenn es eine auch langfristig gleich wirksame, aber weniger riskante Alternative gibt?"

(spa) I

II Symposium im Rahmen der ADA-Jahrestagung 28. Juni 2010 in Orlando (Veranstalter: MSD Sharp \& Dohme) 